\title{
Cooperation-Based Modeling of Sustainable Development: An Approach from Filippov's Systems
}

\author{
Jorge A. Amador $\left(\mathbb{D},{ }^{1}\right.$ Johan Manuel Redondo $\left(\mathbb{D},{ }^{2}\right.$ Gerard Olivar-Tost $\mathbb{D}^{3},{ }^{3}$ \\ and Christian Erazo ${ }^{4}{ }^{4}$ \\ ${ }^{1}$ Instituto de Investigación de Recursos Biológicos Alexander von Humboldt, Bogota, DC, Colombia \\ ${ }^{2}$ Facultad de Ciencias Económicas y Administrativas, Universidad Católica de Colombia, Bogota, DC, Colombia \\ ${ }^{3}$ Departamento de Ciencias Naturales y Tecnología, Universidad de Aysén, Aysen, Chile \\ ${ }^{4}$ Facultad de Ingeniería Mecánica, Electrónica y Biomédica, Universidad Antonio Nariño, Bogota, DC, Colombia
}

Correspondence should be addressed to Gerard Olivar-Tost; gerard.olivar@uaysen.cl

Received 28 April 2021; Revised 4 August 2021; Accepted 9 August 2021; Published 27 September 2021

Academic Editor: Jorge-Antonio Lopez-Renteria

Copyright (c) 2021 Jorge A. Amador et al. This is an open access article distributed under the Creative Commons Attribution License, which permits unrestricted use, distribution, and reproduction in any medium, provided the original work is properly cited.

\begin{abstract}
The concept of Sustainable Development has given rise to multiple interpretations. In this article, it is proposed that Sustainable Development should be interpreted as the capacity of territory, community, or landscape to conserve the notion of well-being that its population has agreed upon. To see the implications of this interpretation, a Brander and Taylor model, to evaluate the implications that extractivist policies have over an isolated community and cooperating communities, is proposed. For an isolated community and through a bifurcation analysis in which the Hopf bifurcation and the heteroclinic cycle bifurcation are detected, 4 prospective scenarios are found, but only one is sustainable under different extraction policies. In the case of cooperation, the exchange between communities is considered by coupling two models such as the one defined for the isolated community, with the condition that their transfers of renewable resources involve conservation policies. Since human decisions do not occur in a continuum, but rather through jumps, the mathematical model of cooperation used is a Filippov System, in which the dynamics could involve two switching manifolds of codimension one and one switching manifold of codimension two. The exchange in the cooperation model, for specific parameter arrangements, exhibits $n$-periodic orbits and chaos. It is notable that, in the cases in which the system shows sliding, it could be interpreted as a recovery delay related to the time needed by the deficit community to recover, until its dependence on the other community stops. It is concluded (1) that a sustainability analysis depends on the way well-being is defined because every definition of well-being is not necessarily sustainable, (2) that sustainability can be visualized as invariant sets in the nonzero region of the space of states (equilibrium points, $n$-periodic orbits, and strange attractors), and (3) that exchange is key to the prevalence of the human being in time. The results question us on whether Sustainable Development is only to keep us alive or if it also implies doing it with dignity.
\end{abstract}

\section{Introduction}

Sustainable Development is a concept that has become relevant [1] since due to the series of criticisms that had been made regarding the global model of economic growth, which put the survival of all living species on the planet at risk, including the human being. Reports such as "Limits to Growth" [2] warned about the capacity of the planet in the face of the dynamics proposed from the socioeconomic point of view to generate growth.
The global impact of the concept did not lead to a homogeneous school of thought on Sustainable Development, but to the establishment of families of conceptual positions that tried to adapt the concept to their interpretations, as in the case of corporate sustainability and environmental sustainability, which made the word sustainability a suffix or the Latin American case in which the language allowed the differentiation between "sostenible" and "sustentable," to eradicate the economic character that the concept was taking on political agendas or its interpretations that gave rise to 
weak/strong sustainability [3], to sustainable landscapes [4] and to the widely recognized approach of Elkington [5], and the triple bottom line is sustainability from social, economic, and environmental dimensions.

The interpretation made in this article of the definition of Sustainable Development proposed by [1]: "satisfying present needs without compromising the satisfaction of the needs of future generations," assumes (1) that the system of needs is not a unique set, but is defined according to the territory, landscape, or community and the ways of life in them, (2) that the system of needs does not have important changes from one generation to another, (3) that satisfying needs has the purpose of generating well-being, and (4) that this well-being must exist for this generation and any future generation. In this sense, sustainability is an emerging expression of the territory, landscape, or community, which results from the interactions of its socio-ecological components, so its analysis must be carried out according to systemic and dynamic form [6]. In this article, then, it will be said that a territory, landscape, or community is sustainable if the notion of well-being that its population has agreed upon is a conservation law and symmetry of time, in the nonnegative region of the space of states.

This interpretation has different implications: (1) if the system of needs depends on the territory, landscape, or community and their ways of life, there cannot be a single sustainability, but there are sustainabilities, (2) if the system of needs can go from one generation to another without important changes, it is because the way in which it is defined has prioritized what is really important, whatever that means, (3) the set of all definitions that could be proposed for well-being would not necessarily lead to Sustainable Development because many of them will only be valid in the short term, and (4) restricting sustainability to the economic, social, and environmental dimensions is insufficient to capture the complexity of a definition of wellbeing that can be perpetuated over time as well as fallacious environmental, social, and economic sustainability considerations that ignore the interdependence that exists between these dimensions and others to make socio-ecological systems viable in the long term.

But the most important implication about well-being, as a conservation law, is that in Sustainable Development wellbeing cannot increase or decrease, unless there are exchanges of information, matter, and energy from one territory, landscape, or community to another, which is completely contrary to the case in which a territory is eroded to guarantee the well-being of another, without compensation for the resources taken being sufficient for its recovery.

Here we study the case in which two socio-ecological systems have exchanges, constituting a new socio-ecological system on which it is not clear how these exchanges will determine their sustainability. In this sense, the purpose of this article is to present the first approach to the study of exchanges between territories, landscapes, and communities within the framework of Sustainable Development from discontinuous piecewise smooth systems and explain the implications of this approach for two communities, based on the analysis of their dynamic behavior.
Due to it is the first approximation, the mathematical model has variables that define a very simple notion of wellbeing, based on populations and available renewable resources, with which it will seek to demonstrate the conservation of well-being.

The mathematical model used for this purpose is a Filippov system $[7,8]$. The choice of this type of system resides in the fact that human decisions do not necessarily occur continuously, but rather through jumps defined by ranges of tolerance to events.

For an introduction to Filippov's systems, see [9-13]. An equivalent formulation in part is found in [14]. For a review of piecewise linear systems, it can be reviewed [15-18]. Regarding the limit cycles in Filippov's systems, it is recommended to review [19]. On the bifurcations of these systems, there are articles from [20-26], together with more specialized articles such as [27-29] for periodic orbits, $[30,31]$ for sliding bifurcations or the Hopf bifurcation compendium of [32]. Other topics that may be of interest are the numerical aspects of the solution of these differential systems [33, 34] or stochastic perturbations to periodic orbits with sliding $[35,36]$.

On the applications of Filippov systems, the works have been mainly oriented to friction oscillators [31, 37-41], neural networks activated by discontinuous functions [42-46], memristor-based neural networks [47-53], neural networks with switching control using the Filippov system with delay [54-57], and electronic converters [58]. On issues related to Sustainable Development, the number of papers is much more limited, with approaches from the analysis of communities [59], from the analysis of companies [60] and others that touch on close issues such as energy systems [61-64], pest or disease control [65-67], HIV behavior $[68,69]$, behavior longterm communities [70], or communications security [71]. It is also worth mentioning a novel approach to the study of systems using multiple switching regions that have been proposed in [72].

For the simulation, tools such as SLIDECONT [73] or smooth solvers [74] have been developed for the analysis of sliding bifurcation of Filippov systems. Numerical continuation methods of these systems have also been proposed [41]. More recently there is the TC-hat software from [75], COCO [76], and MAMBO [77].

The rest of the paper is structured as follows. After this introductory section, two sections are presented in which (1) the effect of the variation of the extraction capacities in an isolated community is modeled and simulated, using a twodimensional continuous model, see Section 2, and (2) the effect of the exchange of resources between two communities, based on a Filippov system, see Section 3. In these models, seeking to have a first approximation of sustainability as conservation of the well-being of territory, landscape, or community, it is assumed that well-being is having renewable resources, which oversimplifies a plausible definition of wellbeing, but allows the presentation of the possibilities of this interpretation of sustainability, as will be seen in the discussion of results' sections, see Section 4, and of conclusions, see Section 5. The article ends with the proposal for future research in this line of work, see Section 6. 


\section{Effect of the Variation of the Extraction Capacities in a Community}

The mathematical model on which this article is based is the one developed by Brander and Taylor [78], who presented a general equilibrium model to represent the dynamic interaction between renewable resources and population, seeking to explain the case of Easter Island.

The Brander and Taylor model has been modified by authors to achieve a better approximation to modern systems of extraction and use of renewable resources, obtaining differential systems of greater dimension and elaboration. For example, multiple economic activities have been incorporated, adding to the extraction of resources and the production of manufactured goods [79] or proposing agriculture as a parallel and different activity to extraction [80]. Institutional adjustments and some economic structures of property rights have also been included, which restrict the conditions of extraction and consumption that could mitigate or dampen the cycles of abundance and famine $[81,82]$ or the consideration of conservation policies that were based on resource extraction charges [83].

Most of the models based on differential equations emerged from the Brander and Taylor model as well as other models of the same type that study the dynamic relationship between population and resources and contemplated isolated societies, without considering migrations or exchanges of information, matter, or energy. However, one can find models with differential equations that somehow incorporate this coupling between societies. For example, in [84], a model is presented that tries to capture the effect that migration has on the degradation of natural resources; in [85], a model is used to investigate the emergent effects of the movement of people, goods, and natural resources, between two societies that have characteristics similar to those of Easter Island; a different coupling method is used in [86], where two new state variables are proposed: the capital inventory and a social development index, for the construction of a dynamic migration network between municipalities of a region in Colombia; finally, in [87], a socio-ecological model of multiple human populations is proposed, which exploit their natural resources or that of another population when their own are scarce, finding that the increase in interacting communities accelerates and aggravates the collapse.

This article, in contrast to [87], studies the long-term effect of economic cooperation between two communities, for which a simplified version of [80] of the Brander and Taylor model was used so that the extraction of resources is considered as the only economic activity developed.

The system of differential equations for the representation of the dynamics of an isolated community considers that the population change is given by the extraction speed that the population $L$ has of its available renewable resources $S$, from a per-capita extraction rate $\epsilon$ [85] and the minimum per-capita caloric requirements $\sigma$ of its population, in consideration of a conversion factor from mass units of the extracted resource to caloric units $\phi$, while for the change in available renewable resources $S$, it is assumed that the renewable resource is regenerated if it is above the $T$ limit (strong growth effect), at a rate of $\rho$ up to that reaches its carrying capacity $K$ (growth limit of the renewable resources) and that depends on the mentioned extraction that the population makes of the resources, as shown in the following equation:

$$
\begin{aligned}
& \frac{\mathrm{d} L}{\mathrm{~d} t}=\phi \varepsilon L S-\sigma L, \\
& \frac{\mathrm{d} S}{\mathrm{~d} t}=\rho S\left(\frac{S}{T}-1\right)\left(1-\frac{S}{K}\right)-\varepsilon L S .
\end{aligned}
$$

System (1) has 4 equilibrium points:

$$
\begin{aligned}
& P_{1}=(0,0), \\
& P_{2}=(0, T), \\
& P_{3}=(0, K), \\
& P_{4}=\left(-\frac{\rho\left(K T \epsilon^{2} \phi^{2}-K \epsilon \phi \sigma-T \epsilon \phi \sigma+\sigma^{2}\right)}{K T \phi^{2} \epsilon^{3}}, \frac{\sigma}{\epsilon \phi}\right) .
\end{aligned}
$$

Following Figure 1, equilibrium $P_{1}$ is always a stable node, and $P_{2}$ is always an unstable saddle-type node, making the Allee effect considered for the resources in the model remarkable. The Allee effect occurs when the regeneration rate slows down at low resource density [88].

When $\varepsilon$ gradually increases, $P_{3}$ and $P_{4}$ collide in what is called a branch point BP. In this collision, $K=\sigma / K \phi$ and $-\rho\left(K T \phi^{2} \epsilon^{2}-K \phi \sigma \epsilon-T \phi \sigma \epsilon+\sigma^{2}\right) / \phi^{2} \epsilon^{3} K T=0$. When solving for $\varepsilon$, we found that $\mathrm{BP}$ occurs at $\varepsilon^{\mathrm{BP}}=\sigma / K \phi$, and if $\varepsilon<\varepsilon^{\mathrm{BP}}$ population is negative and $P_{4}$ is unstable while $P_{3}$ is stable, equilibrium population is positive and $P_{4}$ becomes a stable focus if $\varepsilon>\varepsilon^{\mathrm{BP}}$ until the Hopf bifurcation $H$ is reached, see Figure 1. To prove the existence of a Hopf bifurcation, we define the Jacobian matrix as

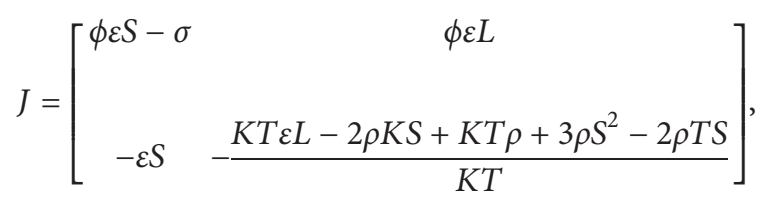

whose eigenvalues are

$$
\lambda_{1,2}=\frac{1}{2}\left(\operatorname{Tr} J \pm \sqrt{\operatorname{Tr} J^{2}-4 \operatorname{Det} J}\right) .
$$

$\operatorname{Tr} J$ corresponds to the trace of $J$ and Det $J$ to its determinant. When solving for $\epsilon$, the trace evaluated at $P_{4}$, we find that a Hopf bifurcation occurs at $\epsilon^{H}=2 \sigma /(K+T) \phi$ since $P_{4}$ has a pair of pure imaginary eigenvalues, satisfying the equilibrium condition, giving rise to the instability of the $P_{4}$ focus. Furthermore, the system undergoes the Hopf bifurcation as long as the real part of eigenvalues really change sign by crossing zero. The previous condition can be proved by demonstrating that the cross speed of the real part with respect to the control parameter is nonzero. Since 


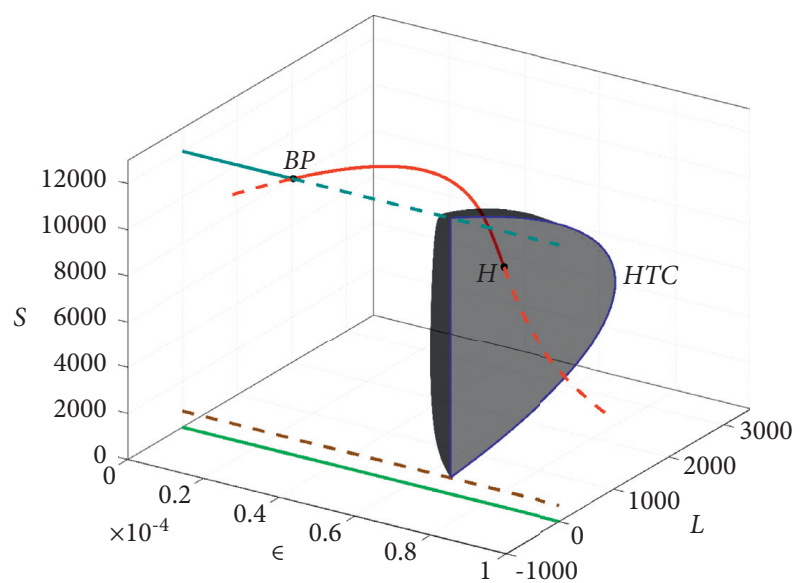

(a)

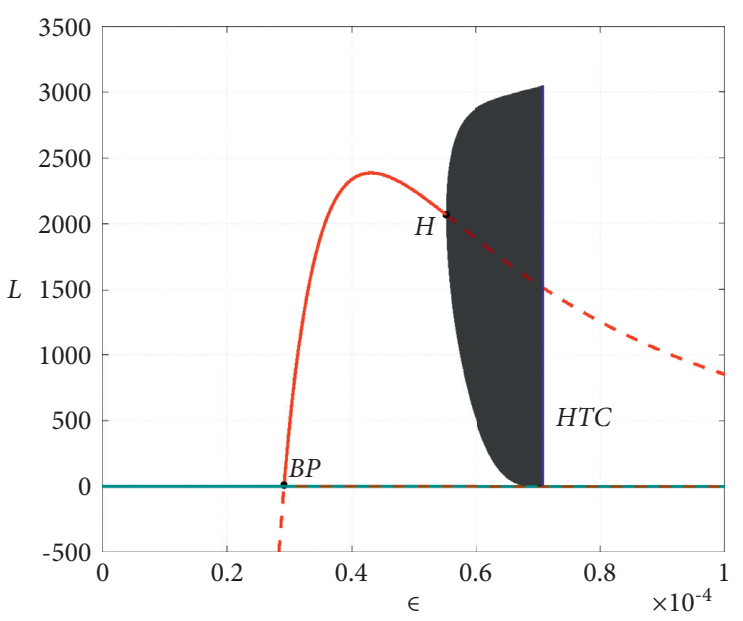

(b)

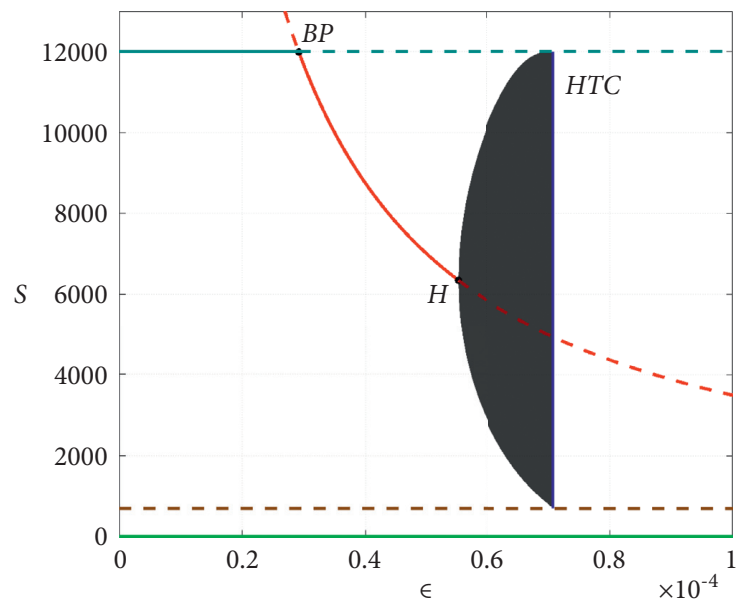

(c)

Figure 1: Steady-state change for an isolated community when the per-capita extraction rate $\epsilon$ is taken as the control parameter. The solid line represents stable equilibrium points, and the dashed line represents unstable equilibrium points. The shaded area represents limit cycles. (a) Bifurcation diagram, (b) projection on $\epsilon-L$ plane, and (c) projection on $\epsilon$-S plane. Parameter values for this simulation are $\sigma=0.14$, $\phi=0.4, K=12000, T=700$, and $\rho=0.03$.

$(1 / 2) \operatorname{Tr} J$ is the real part of the eigenvalues, the cross speed is given by

$$
\frac{\mathrm{d}}{\mathrm{d} \epsilon}[\operatorname{Re}(\lambda(\epsilon))]_{\epsilon=\epsilon^{H}}=\frac{\phi \rho(K+T)^{3}}{8 \sigma K T} \neq 0,
$$

so that the transversality condition is satisfied. Finally, according to the sign of the first Lyapunov coefficient, $l_{1}=-1.675825 e-07$, and the Hopf bifurcation is supercritical giving rise to a stable limit cycle [89]. Numerical continuation of periodic orbits and detection of heteroclinic bifurcation HTC were developed using MATCONT [90].

On the contrary, the nodes $P_{3}$ and $P_{4}$ collide at $\varepsilon \approx 0.3$, making the node $P_{3}$, related to load capacity, go from stable to unstable (chair type), while node $P_{4}$ makes it from unstable to stable, for a positive increment of $\epsilon$.

At $\varepsilon \approx 0.55$ a Hopf bifurcation occurs, giving rise to the instability of the $P_{4}$ node, which becomes a repulsive node, while an attractor limit cycle emerges, that becomes large, for positive increases of $\epsilon$, until in $\epsilon \approx 0.7$, it gives rise to heteroclinic orbits between the nodes $P_{2}$ and $P_{3}$, forming a heteroclinic cycle, from which the limit cycle disappears.

Note that, before the branch point BP, the amount of resources extracted does not meet the population's need, leading to its disappearance. From BP and up to the Hopf bifurcation $H$, the resources extracted are sufficient to sustain a specific amount of population, gradually decreasing the support capacity of the system. Between $H$ and the heteroclinic bifurcation HTC, the population-resources relationship enters into a dynamic of oscillation between scarcity and abundance, which becomes critical when the HTC is exceeded, at which time, followed by a moment at maximum abundance, the population will grow so large that it will critically deplete resources, leading to the collapse of the population and its resources. 


\section{Effect of Resource Exchange between Two Communities}

In this section, the dynamics of exchange between two communities for the supply of their population's needs are considered, in which decision-makers are willing to enforce rules on resource extraction, have a conservation policy, and have a complete understanding of the socio-ecological system that allows them to define clear extraction limits.

3.1. Exchange with Continuous Coupling. The model for two communities, see system (6), assumes that each population keeps the proportion $C_{i}, i=1,2$, while exchanging the proportion $\left(1-C_{i}\right)$ of the resources that produces but also protects an amount equal to its unextracted resources to the amount received by the exchange $S_{i \longrightarrow j}, i=1,2, i \neq j$, in caloric units:

$$
\begin{aligned}
& \frac{\mathrm{d} L_{1}}{\mathrm{~d} t}=C_{1} \phi_{1} \varepsilon_{1} L_{1} S_{1}+\left(1-C_{2}\right) \phi_{2} \varepsilon_{2} L_{2} S_{2}-\sigma_{1} L_{1}, \\
& \frac{\mathrm{d} S_{1}}{\mathrm{~d} t}=\rho_{1} S_{1}\left(\frac{S_{1}}{T_{1}}-1\right)\left(1-\frac{S_{1}}{K_{1}}\right)-\varepsilon_{1} L_{1}\left(S_{1}-S_{2 \longrightarrow 1}\right), \\
& \frac{\mathrm{d} L_{2}}{\mathrm{~d} t}=C_{2} \phi_{2} \varepsilon_{2} L_{2} S_{2}+\left(1-C_{1}\right) \phi_{1} \varepsilon_{1} L_{1} S_{1}-\sigma_{2} L_{2}, \\
& \frac{\mathrm{d} S_{2}}{\mathrm{~d} t}=\rho_{2} S_{2}\left(\frac{S_{2}}{T_{2}}-1\right)\left(1-\frac{S_{2}}{K_{2}}\right)-\varepsilon_{2} L_{2}\left(S_{2}-S_{1 \longrightarrow 2}\right) .
\end{aligned}
$$

The resource conservation policy is given by equation (7), which is a conversion factor between the resources of the two communities:

$$
S_{i \longrightarrow j}=\frac{\left(1-C_{i}\right) \phi_{i} \epsilon_{i} S_{i}}{\phi_{j} \epsilon_{j}} .
$$

For simplicity, simulations have been carried out with normalized $S_{i}$, considering $s_{i}=S_{i} / K_{i}$.

3.2. Exchange Rules between Communities. For the representation of resources' exchange rules between communities, it was considered that the exchange proportions $C_{i}$ depend on levels of resources that are defined for each community, understanding that a level is a set of states of available renewable resources, such that, if the level between the communities is different, there is an exchange from the community with greater resources to the community with fewer resources, and if the level is the same, no exchanges are made. This representation implies that the exchange rates $C_{i}$ change according to the available level of resources that the two communities have at each instant of time.

In this way, it is assumed that cooperation is a decision that is made mutually under certain considerations related to resource stocks, whose main objective is to mitigate the overexploitation of natural resources of the needy community (understand a needy community as one that has brought its available renewable resources to a deficit threshold).
The 4-dimensional space of states has two switching regions $\Sigma_{i}, i=1,2$, each of which defines the decision change limits, also defining two levels of available renewable resources by community: deficit and surplus. Considering that the communities would not be willing to have their levels defined differently to avoid exposing their resources in the exchange measures, it has been considered that the levels of each community have the same thresholds.

3.3. Behavior with Exchange Rules through Filippov Systems. To represent the rules defined above and taking into account that they define a discontinuous system, we will now present the formalism of Filippov's systems [8] required to obtain the differential system that models the exchange between two communities.

3.3.1. Formalism of Filippov's Systems. In Filippov systems with a commutation surface, the state space is divided into two regions $R_{1}$ and $R_{2}$ through a surface $\Sigma$, defined as the set 0 of a smooth scalar function $h$. Thus, we have

$$
\dot{x}=f(x)=\left\{\begin{array}{ll}
f_{1}(x), & x \in R_{1}, \\
f_{2}(x), & x \in R_{2},
\end{array}, x(0)=x_{0} \in \mathbb{R}^{n},\right.
$$

where $f_{1}$ and $f_{2}$ are smooth vector fields. The regions $R_{1}$ and $R_{2}$ and the surface $\Sigma$ are defined as

$$
\begin{aligned}
R_{1} & =\left\{x \in \mathbb{R}^{n}: h(x)>0\right\}, \\
R_{2} & =\left\{x \in \mathbb{R}^{n}: h(x)<0\right\}, \\
\Sigma & =\left\{x \in \mathbb{R}^{n}: h(x)=0\right\} .
\end{aligned}
$$

In this case, the dynamics on $\Sigma$ are classified in

(i) Sliding Region. $\stackrel{\wedge}{\Sigma}=\left\{x \in \mathbb{R}^{n}: \mathscr{L}_{f_{1}} h(x) \cdot \mathscr{L}_{f_{2}} h(x)\right.$ $\left.<0, \mathscr{L}_{f_{1}} h(x)<0\right\}, \hat{\Sigma} \subset \Sigma$, when both vector fields $f_{1}(x)$ and $f_{2}(x)$ point to $\Sigma$

(ii) Escaping Region. $\Sigma_{e}=\left\{x \in \mathbb{R}^{n}: \mathscr{L}_{f_{1}} h(x) \cdot \mathscr{L}_{f_{2}} h(x)\right.$ $\left.<0, \mathscr{L}_{f_{1}} h(x)>0\right\}, \Sigma_{e} \subset \Sigma$

(iii) Crossing Region. $\Sigma_{c}=\left\{x \in \mathbb{R}^{n}: \mathscr{L}_{f_{1}} h(x) \cdot \mathscr{L}_{f_{2}} h\right.$ $(x)>0\}, \Sigma_{c} \subset \Sigma$

Here, $\mathscr{L}_{f_{1,2}} h(x):=\nabla h(x) \cdot f_{1,2}(x)$ is defined as the Lie derivative of $h(x)$ with respect to the vector field $f_{1,2}$. Once the evolution of the system reaches the sliding surface, the dynamics can be defined as a linear combination of the vector fields $\left(f_{1}\right.$ and $\left.f_{2}\right)$ through the Filippov formalism [8], namely,

$$
\dot{x}=f_{\Sigma}(x), \quad x \in \hat{\Sigma},
$$

where

$$
f_{\Sigma}=(1-\lambda) f_{1}(x)+\lambda f_{2}(x),
$$

and $\lambda$ is the solution of

$$
\left\{\begin{array}{l}
0=\nabla h(x)^{T} \cdot f_{\Sigma}(x) \\
0=h(x)
\end{array}\right.
$$


On the contrary, the trajectory of the system leaves the sliding region as soon as the vector fields $f_{1,2}$ become tangent to $\Sigma$, that is, making $\lambda=0$ and $\lambda=1$, and we obtain $\mathscr{L}_{f_{1}} h(x)=0$ or $\mathscr{L}_{f_{2}} h(x)=0$.

Now, if we consider a system with two commutation surfaces $\Sigma_{1}$ and $\Sigma_{2}$, according to Filippov's formalism, the state space will be divided into four regions $R_{1}, R_{2}, R_{3}$, and $R_{4}$ :

$$
\begin{array}{ll}
R_{1}: f_{1} \text { for } & h_{1}>0, h_{2}>0, \\
R_{2}: f_{2} \text { for } & h_{1}<0, h_{2}>0, \\
R_{3}: f_{3} \text { for } & h_{1}<0, h_{2}<0, \\
R_{4}: f_{4} \text { for } & h_{1}>0, h_{2}<0 .
\end{array}
$$

In this case, the trajectories of the system can evolve in any of the mentioned regions as well as on the regions $\Sigma_{1}$ and $\Sigma_{2}$. Additionally, the system can evolve on the commutation surface of codimension two, defined by the intersection of the two commutation surfaces of codimension one (i.e., $\left.\Sigma=\Sigma_{1} \cap \Sigma_{2}\right)$ :

$$
\Sigma=\left\{x \in \mathbb{R}^{n}: h(x)=0, h(x)=\left[\begin{array}{l}
h_{1}(x) \\
h_{2}(x)
\end{array}\right]\right\} .
$$

Now, the codimension 1 sliding vector fields are defined as $f_{\Sigma}^{ \pm}$with respect to each switching surface $\Sigma_{1,2}^{ \pm}$, i.e.,

$$
\begin{aligned}
& \dot{x}=f_{\Sigma_{1}^{+}}=\left(1-\lambda_{1}\right) f_{1}(x)+\lambda_{1} f_{4}(x), \lambda_{1}=\frac{\mathscr{L}_{f_{1}} h_{1}(x)}{\mathscr{L}_{f_{1}-f_{4}} h_{1}(x)}, \\
& \dot{x}=f_{\Sigma_{1}^{-}}=\left(1-\lambda_{2}\right) f_{2}(x)+\lambda_{2} f_{3}(x), \lambda_{2}=\frac{\mathscr{L}_{f_{2}} h_{1}(x)}{\mathscr{L}_{f_{2}-f_{3}} h_{1}(x)}, \\
& \dot{x}=f_{\Sigma_{2}^{+}}=\left(1-\lambda_{3}\right) f_{1}(x)+\lambda_{3} f_{2}(x), \lambda_{3}=\frac{\mathscr{L}_{f_{1}} h_{2}(x)}{\mathscr{L}_{f_{1}-f_{2}} h_{2}(x)}, \\
& \dot{x}=f_{\Sigma_{2}^{-}}=\left(1-\lambda_{4}\right) f_{3}(x)+\lambda_{4} f_{4}(x), \lambda_{4}=\frac{\mathscr{L}_{f_{3}} h_{2}(x)}{\mathscr{L}_{f_{3}-f_{4}} h_{2}(x)} .
\end{aligned}
$$

If a path is followed on one of the sliding surfaces $\sum_{1,2}^{ \pm}$, the attractiveness can be characterized according to the firstorder output conditions (tangency conditions). Whenever $\lambda_{1,2,3,4}=0$ and $\lambda_{1,2,3,4}=1$, the exit conditions of the first order are expressed by

$$
\begin{aligned}
& \mathscr{L}_{f_{1}} h_{1}(x)=0, \\
& \mathscr{L}_{f_{2}} h_{1}(x)=0, \\
& \mathscr{L}_{f_{1}} h_{2}(x)=0, \\
& \mathscr{L}_{f_{2}} h_{2}(x)=0, \\
& \mathscr{L}_{f_{3}} h_{1}(x)=0, \\
& \mathscr{L}_{f_{4}} h_{1}(x)=0, \\
& \mathscr{L}_{f_{3}} h_{2}(x)=0, \\
& \mathscr{L}_{f_{4}} h_{2}(x)=0 .
\end{aligned}
$$

The vector field of sliding on the commutation surface $\Sigma$ can be defined through the convex method of Filippov [8]. In this way, the dynamics on $\Sigma$ are

$$
F_{\Sigma}(x)=\sum_{i=1}^{4} \lambda_{i}(x) f_{i}(x)
$$

where

$$
\lambda_{i}(x) \geq 0 \wedge \sum_{i=1}^{4} \lambda_{i}(x)=1,
$$

taking into account that $F_{\Sigma}(x)$ must be tangent to $\Sigma$, that is, $\mathscr{L}_{F_{\Sigma}} h_{i}(x)=0, i=1,2$. Clearly, it can be seen from the above that there is no single solution for the coefficients $\lambda_{i}(x)$ since we now have a system of three equations with four unknowns. In the literature, two systematic methods allow defining the codimension 2 sliding vector field under certain conditions, the bilinear combination [91] and the method called moments of solutions [92] although the ambiguity that arises when considering multiple switching surfaces remains a problem under study. A nonlinear formulation of the sliding vector field called hidden dynamics is proposed in [93], which has been effective in modeling real mechanical phenomena such as friction.

The bilinear interpolation method was originally introduced by Seidman [91] and further studied in [92]. The idea is to obtain a vector field on the intersection by forming a bilinear interpolation among the four vector fields:

$$
f_{B}:=(1-\hat{\alpha})(1-\hat{\beta}) f_{1}+(1-\hat{\alpha}) \hat{\beta} f_{2}+\hat{\alpha}(1-\hat{\beta}) f_{3}+\hat{\alpha} \hat{\beta} f_{4}
$$

where $\hat{\alpha}$ and $\hat{\beta}$ are smooth functions of $x \in \Sigma$, between $[0,1]$. Under the orthogonality conditions $\left(\mathscr{L}_{F_{B}}\left(h_{1}\right)(x)=\right.$ $\left.\mathscr{L}_{F_{B}}\left(h_{2}\right)(x)=0\right), \hat{\alpha}$ and $\hat{\beta}$ can be found by solving the following nonlinear system equations:

$$
\begin{gathered}
(1-\hat{\alpha})(1-\hat{\beta})\left[\begin{array}{l}
\mathscr{L}_{f_{1}} h_{1} \\
\mathscr{L}_{f_{1}} h_{2}
\end{array}\right]+(1-\hat{\alpha}) \hat{\beta}\left[\begin{array}{l}
\mathscr{L}_{f_{2}} h_{1} \\
\mathscr{L}_{f_{2}} h_{2}
\end{array}\right] \\
+\hat{\alpha}(1-\hat{\beta})\left[\begin{array}{l}
\mathscr{L}_{f_{3}} h_{1} \\
\mathscr{L}_{f_{3}} h_{2}
\end{array}\right]+\hat{\alpha} \hat{\beta}\left[\begin{array}{l}
\mathscr{L}_{f_{4}} h_{1} \\
\mathscr{L}_{f_{4}} h_{2}
\end{array}\right]=0 .
\end{gathered}
$$

Assuming that we are following a trajectory on $\Sigma$, the second-order conditions are defined by

$$
\begin{gathered}
\mathscr{L}_{f} \sum_{1}^{+} h_{1}(x)=0, \\
\mathscr{L}_{f} \sum_{1}^{-} h_{1}(x)=0, \\
\mathscr{L}_{f} \sum_{2}^{+} h_{2}(x)=0, \\
\mathscr{L}_{f} \sum_{2}^{-} h_{2}(x)=0 .
\end{gathered}
$$

3.3.2. Filippov System Model. The variation in the exchange rates of each community is assumed according to the following rule: 


$$
\begin{aligned}
& C_{1}= \begin{cases}C_{1}^{+}, & \text {if } h_{1}>0, \\
C_{1}^{-}, & \text {if } h_{1}<0,\end{cases} \\
& C_{2}= \begin{cases}C_{2}^{+}, & \text {if } h_{2}>0, \\
C_{2}^{-}, & \text {if } h_{2}<0 .\end{cases}
\end{aligned}
$$

Based on the Filippov formalism, the dynamics of resource exchange between two communities can be rewritten in state space by making $x=\left[L_{1}, S_{1}, L_{2}, S_{2}\right]^{T}, h_{1}=S_{1}-\alpha_{T}$, and $h_{2}=S_{2}-\alpha_{T}, \alpha_{T}$ being the decision threshold for both communities, that is,

$$
\dot{x}=f(x)= \begin{cases}f_{1}(x), & x \in R_{1} \\ f_{2}(x), & x \in R_{2} \\ f_{3}(x), & x \in R_{3} \\ f_{4}(x), & x \in R_{4}\end{cases}
$$

where

$$
\begin{aligned}
& f_{1}(x)=\left(\begin{array}{c}
C_{1}^{+} \phi_{1} \epsilon_{1} L_{1} S_{1}-\sigma_{1} L_{1}+\left(1-C_{2}^{+}\right) \phi_{2} \epsilon_{2} L_{2} S_{2} \\
\rho_{1} S_{1}\left(\frac{S_{1}}{T_{1}}-1\right)\left(1-\frac{S_{1}}{K_{1}}\right)-\epsilon_{1} L_{1}\left(S_{1}-S_{2 \longrightarrow 1}\right) \\
C_{2}^{+} \phi_{2} \epsilon_{2} L_{2} S_{2}-\sigma_{2} L_{2}+\left(1-C_{1}^{+}\right) \phi_{1} \epsilon_{1} L_{1} S_{1} \\
\rho_{2}\left(\frac{S_{2}}{T_{2}}-1\right)\left(1-\frac{S_{2}}{K_{2}}\right) S_{2}-\epsilon_{2} L_{2}\left(S_{2}-S_{1 \longrightarrow 2}\right)
\end{array}\right), \\
& f_{2}(x)=\left(\begin{array}{c}
C_{1}^{-} \phi_{1} \epsilon_{1} L_{1} S_{1}+\left(1-C_{2}^{+}\right) \phi_{2} \epsilon_{2} L_{2} S_{2}-\sigma_{1} L_{1} \\
\rho_{1} S_{1}\left(\frac{S_{1}}{T_{1}}-1\right)\left(1-\frac{S_{1}}{K_{1}}\right)-\epsilon_{1} L_{1}\left(S_{1}-S_{2 \longrightarrow 1}\right) \\
C_{2}^{+} \phi_{2} \epsilon_{2} L_{2} S_{2}+\left(1-C_{1}^{-}\right) \phi_{1} \epsilon_{1} L_{1} S_{1}-\sigma_{2} L_{2} \\
\rho_{2} S_{2}\left(\frac{S_{2}}{T_{2}}-1\right)\left(1-\frac{S_{2}}{K_{2}}\right)-\epsilon_{2} L_{2}\left(S_{2}-S_{1 \longrightarrow 2}\right)
\end{array}\right), \\
& f_{3}(x)=\left(\begin{array}{c}
C_{1}^{-} \phi_{1} \epsilon_{1} L_{1} S_{1}+\left(1-C_{2}^{-}\right) \phi_{2} \epsilon_{2} L_{2} S_{2}-\sigma_{1} L_{1} \\
\rho_{1} S_{1}\left(\frac{S_{1}}{T_{1}}-1\right)\left(1-\frac{S_{1}}{K_{1}}\right)-\epsilon_{1} L_{1}\left(S_{1}-S_{2 \longrightarrow 1}\right) \\
C_{2}^{-} \phi_{2} \epsilon_{2} L_{2} S_{2}+\left(1-C_{1}^{-}\right) \phi_{1} \epsilon_{1} L_{1} S_{1}-\sigma_{2} L_{2} \\
\rho_{2} S_{2}\left(\frac{S_{2}}{T_{2}}-1\right)\left(1-\frac{S_{2}}{K_{2}}\right)-\epsilon_{2} L_{2}\left(S_{2}-S_{1 \longrightarrow 2}\right)
\end{array}\right), \\
& f_{4}(x)=\left(\begin{array}{c}
C_{1}^{+} \phi_{1} \epsilon_{1} L_{1} S_{1}+\left(1-C_{2}^{-}\right) \phi_{2} \epsilon_{2} L_{2} S_{2}-\sigma_{1} L_{1} \\
\rho_{1} S_{1}\left(\frac{S_{1}}{T_{1}}-1\right)\left(1-\frac{S_{1}}{K_{1}}\right)-\epsilon_{1} L_{1}\left(S_{1}-S_{2 \longrightarrow 1}\right) \\
C_{2}^{-} \phi_{2} \epsilon_{2} L_{2} S_{2}+\left(1-C_{1}^{+}\right) \phi_{1} \epsilon_{1} L_{1} S_{1}-\sigma_{2} L_{2} \\
\rho_{2} S_{2}\left(\frac{S_{2}}{T_{2}}-1\right)\left(1-\frac{S_{2}}{K_{2}}\right)-\epsilon_{2} L_{2}\left(S_{2}-S_{1 \longrightarrow 2}\right)
\end{array}\right)
\end{aligned}
$$

Furthermore, the switching manifold is defined as

$$
\begin{aligned}
& \Sigma_{1}=\left\{x \in \mathbb{R}^{4}: S_{1}-\alpha_{T}=0\right\}, \\
& \Sigma_{2}=\left\{x \in \mathbb{R}^{4}: S_{2}-\alpha_{T}=0\right\} .
\end{aligned}
$$

It is worth to mention that, due to parameter configuration, the flow does not enter into switching manifold of codimension two, and it can be seen in Section 3.4.1 for more details. By solving $f_{\Sigma_{1,2}}^{ \pm}=0$, we found that there exists an equilibrium point outside the sliding region, and thus, the system does not have pseudoequilibrium points. Analytical expressions of tangency points are

$$
\begin{aligned}
& \mathscr{L}_{f_{1}} h_{1}(x)=\rho_{1} S_{1}\left(\frac{S_{1}}{T_{1}}-1\right)\left(1-\frac{S_{1}}{K_{1}}\right)-\epsilon_{1} L_{1}\left(S_{2}-\frac{\left(1-C_{2}^{+}\right) \phi_{2} \epsilon_{2} S_{2}}{\phi_{1} \epsilon_{1}}\right)=0, \\
& \mathscr{L}_{f_{4}} h_{1}(x)=\rho_{1} S_{1}\left(\frac{S_{1}}{T_{1}}-1\right)\left(1-\frac{S_{1}}{K_{1}}\right)-\epsilon_{1} L_{1}\left(S_{2}-\frac{\left(1-C_{2}^{-}\right) \phi_{2} \epsilon_{2} S_{2}}{\phi_{1} \epsilon_{1}}\right)=0, \\
& \mathscr{L}_{f_{1}} h_{2}(x)=\rho_{2} S_{2}\left(\frac{S_{2}}{T_{2}}-1\right)\left(1-\frac{S_{2}}{K_{2}}\right)-\epsilon_{2} L_{2}\left(S_{2}-\frac{\left(1-C_{1}^{+}\right) \phi_{1} \epsilon_{1} S_{1}}{\phi_{2} \epsilon_{2}}\right)=0, \\
& \mathscr{L}_{f_{2}} h_{2}(x)=\rho_{2} S_{2}\left(\frac{S_{2}}{T_{2}}-1\right)\left(1-\frac{S_{2}}{K_{2}}\right)-\epsilon_{2} L_{2}\left(S_{2}-\frac{\left(1-C_{1}^{-}\right) \phi_{1} \epsilon_{1} S_{1}}{\phi_{2} \epsilon_{2}}\right)=0 .
\end{aligned}
$$

Because $\alpha_{T}$ is the decision threshold for both communities, then we can replace $S_{1}$ and $S_{2}$ in the above equations since it is supposed that the decision-making threshold is equal in both communities. Next, we can solve for $L_{1}$ and $L_{2}$ to graph sliding regions, see the yellow región in Figure 2.

3.4. Dynamics of Exchange between Communities with One Decision Level. From the system defined by the vector fields presented in equations (24)-(27), we will present the results of the simulations carried out under the following conditions, which represent the exchange policy between the two communities:

(i) Similar parameter configurations are considered between the two communities that exchange their resources, except in the case of the effective extraction rate and regeneration rate of renewable resources of each community

(ii) It is assumed that the effective rate of extraction of the second community $\epsilon_{2}$ is high in consideration of that defined for the first community so that, by depleting its resources more quickly, it does not count on the cooperation of the community one, and it will collapse and disappear (as in the case of high extraction of resources from the isolated community presented in Section 2).

(iii) It is assumed that the renewable resources of each community are regenerated at different rates, that is, $\rho_{1} \neq \rho_{2}$. This is to suggest that the physiographic, climatic, and productive conditions of the two communities are not necessarily the same, and each one has its complexity.

(iv) It is assumed that the switching regions that define the decision thresholds of the communities are in the same percentage value of resources since they are 


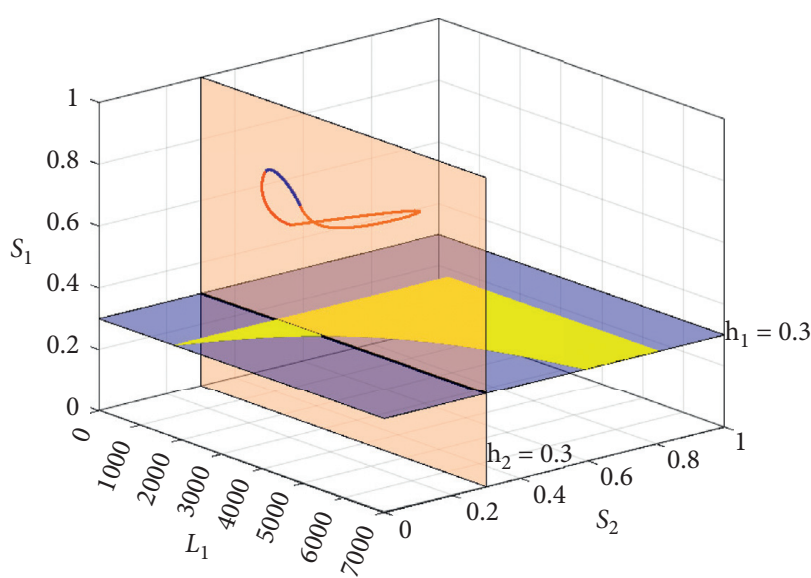

(a)

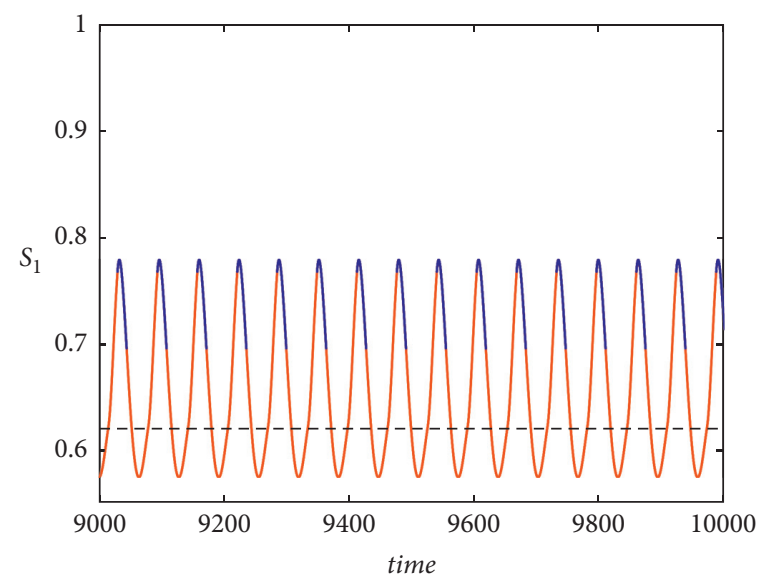

$-\mathrm{C}_{2}$
-- Isolated

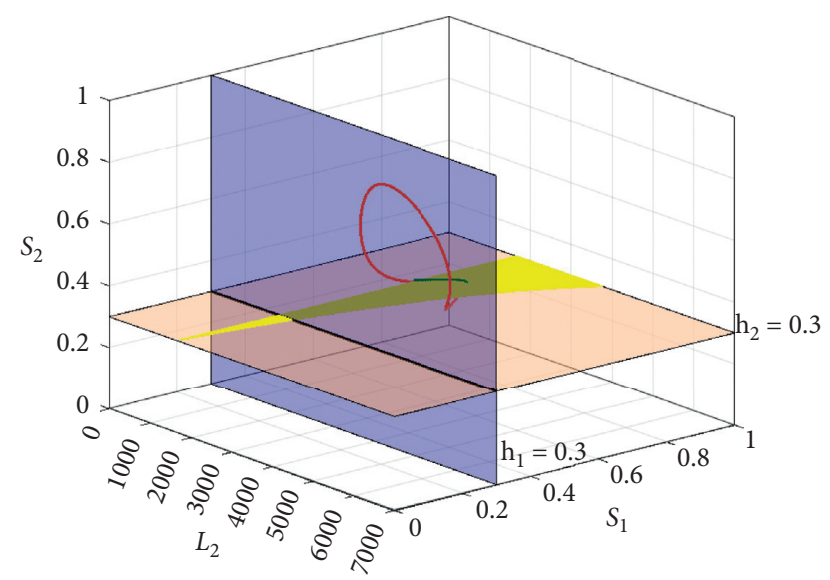

(b)

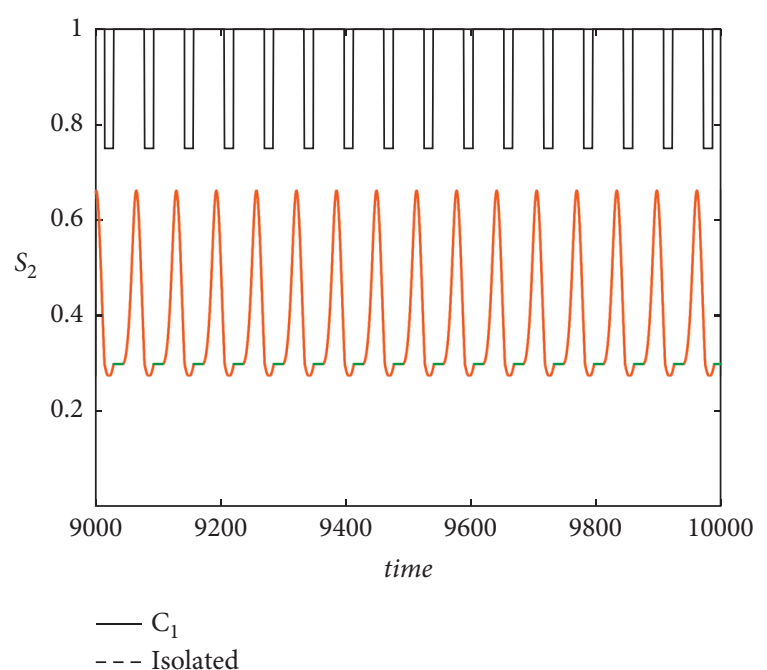

(d)

(c)

Figure 2: Periodic behavior induced by switching regions for $\epsilon_{1}=4.7 e^{-5}$.

framed in the same collaboration policy, guaranteeing that their exchanges occur under equal conditions.

(v) Whenever a community is at the deficit threshold, the collaboration policy says that the community at the surplus threshold will cooperate with up to a certain percentage of its resources, indicating that cooperation only occurs in one direction.

(vi) If the two communities are at the same threshold, the exchanges $C_{1}=C_{2}=1$ are closed, leaving them as if they were isolated communities. In the case where they are at the surplus threshold, the exchange would not be necessary, while in the case where they are at the deficit threshold, the exchange would not be probable since the communities would be putting at risk the little resource that they have left to sustain their populations.

The initial condition of the simulations is found in the surplus thresholds for the two communities so that there is room to show resource management errors, represented in their extraction measures in contrast to the resource regeneration capacity and how a policy of cooperation based on exchange becomes an alternative for the community on the threshold of deficit.

The simulations presented show the results for parameter arrangements that led to obtaining periodic and chaotic behaviors (see Section 3.4.2), which are verified in the projections of the steady-state diagram presented in Section 3.4.3.

The general parameter values used in these simulations are $\sigma_{i}=0.14, \rho_{1}=0.03, \rho_{2}=0.04, T_{i}=700, K_{i}=12000$, $\epsilon_{2}=7 e^{-5}$, and $\phi_{i}=0.4$. The initial condition considered was $\left(L_{1}, S_{1}, L_{2}, S_{2}\right)=(2000,10400,2500,8600)$, the values for the exchange decision through the switching regions were set at $30 \%$, and the exchange rate defined when the community $i$ cooperates with the other was $25 \%$ (i.e., $\left.1-C_{i}=0.25, i=1.2\right)$.

The effective extraction rate $\epsilon_{1}$ is taken as a control parameter to perform the steady-state analysis presented in Section 3.4.3. With this configuration, it is avoided to reach the switching manifold of codimension two, which corresponds to the intersection between the two switching manifolds $\Sigma_{i}$ of codimension one, described in equation (14). 
To present the simulations of this 4-dimensional system, 3-dimensional projections were used to allow the switching manifolds to be adequately visualized. It should be understood then that the switching regions will appear with a smaller dimension than they have. This was achieved by using the spaces $s_{1}-L_{1}-s_{2}$ and $s_{2}-L_{2}-s_{1}$. Also, it was considered that the colors facilitate the reading of the simulations. The three-dimensional projection of the switching region $\Sigma_{1}$ at $h_{1}=0.3$ is colored blue, while the region $\Sigma_{2}$ at $h_{2}=0.3$ is colored orange. The sliding regions were drawn in yellow. The trajectory in orange corresponds to that defined by the vector fields $f_{i}$, in what we have called the "thresholds," while in blue and green, to contrast with the colors of the switching regions and the projections of the landslide trajectories.

The corresponding time series of the resource variables $s_{i}$ and the signal of the exchange variable $C_{i}$ were added to the three-dimensional projections, considering that (1) the solution for the case of communities is drawn on a continuous line coupled, (2) the dotted line shows the trajectory of the case of communities that evolve in isolation $C_{i}=1$, and (3) the colors correspond to the colors used in the three-dimensional projection.

3.4.1. Periodic Behavior. For the first simulation, it has been considered to compare the behavior between two communities under the effects of two scenarios: (1) noncooperation and (2) cooperation, considering a relatively low extraction rate from community one $\epsilon_{1}$, see Figure 2 .

In the noncooperation scenario, presented through dotted lines in the time series of Figures 2(c) and 2(d), it is shown how the first community has reached a nonzero equilibrium value of its resources, while the second has disappeared.

In the cooperation scenario, a 1-periodic behavior is obtained with sliding over the switching manifold of community two $\Sigma_{2}$, which means that, through the exchange of resources, community two no longer disappears, achieving that the two communities are preserved in time, through a periodic cycle, see Figures 2(a) and 2(b).

The black signal, in Figures 2(c) and 2(d), shows the behavior of exchanges between communities; while community 1 does not receive resources $\left(C_{2}=1\right)$, community two does through periodic pulses that satisfy their noncontinuous needs $\left(0.75<C_{1}<1\right)$.

In this way, due to the configuration of the system, we see that the management of the socio-ecological system shown by community one, in scenarios of cooperation or noncooperation, allows it to be maintained over time, while community two only achieves it through cooperation.

The exchange avoids collapse and leads to a process of recovery of the community in deficit, which is not immediate, since it must overcome a delay that occurs while sliding over the switching manifold, possibly related to the recovery of its resources and education for cultural transformation and sustainable management, to finally appreciate recovery and stop dependence on the other community.
3.4.2. Chaotic Behavior. For the second simulation, again, it has been considered to compare the behavior between the two communities under the scenarios of (1) noncooperation and (2) cooperation, but now a relative extraction rate of the one $\epsilon_{1}$ community has been considered high, see Figure 3 .

In the noncooperation scenario, which is shown through dotted lines in the time series of Figures 3(c) and 3(d), isolated communities obtain opposite effects; the first community reaches the equilibrium of its resources despite having considerably increased its resource extraction rate $\epsilon_{1}$, while the second community disappears.

In the cooperation scenario, chaotic behavior is obtained with sliding over the switching manifold of community two $\Sigma_{2}$, which means that, through the exchange of resources, community two no longer disappears, achieving that the two communities are preserved in time, with their trajectory confined to a region of state space, see Figures 3(a) and 3(b).

The black signal, in Figures 3(c) and 3(d), shows the behavior of exchanges between communities; while community 1 remains without receiving resources $\left(C_{2}=1\right)$, community two continues depending on the other community, but now, through irregular pulses that satisfy their noncontinuous needs $\left(0.75<C_{1}<1\right)$.

Under this system configuration, the management of the socio-ecological system shown by community one, in scenarios of cooperation or noncooperation, again allows it to be maintained over time, while community two only achieves it through cooperation.

In the cooperation scenario, for the extraction value relatively higher than the one used in the periodic system configuration of Section 3.4.1, the exchange once again avoids collapse and leads to a process of recovery of the community in deficit but this implies that the contributing community enters into a chaotic behavior confined to states that do not expose their resources and that the receiving community oscillates between the thresholds of surplus and deficit, achieving this long in the first. It draws the attention of community two that, leaving the deficit threshold, they must slide in the switching manifold during irregular (chaotic) periods that perhaps express different levels of consciousness, although their extraction policy is the same.

3.4.3. Steady-State Analysis. In this final simulation, a set of diagrams containing the steady-state mappings are presented, obtained for each of the state variables by the variation of the parameter $\epsilon_{1}$ in the range of values where resource exchanges occur between the communities, involving sliding over the switching manifold $\Sigma_{2}$.

For the elaboration of this diagram, a total of 3000 simulations were carried out from an initial condition centered on $\left(L_{1}, S_{1}, L_{2}, S_{2}\right)=(2000,10400,2500,8600)$, in which each simulation took a value of the $\epsilon_{1}$ parameter in the range of $4.6 \times 10^{-5}$ to $5.5 \times 10^{-5}$. This range of the control parameter was chosen for convenience, for two reasons: (1) it is the range in which sliding occurs over the switching manifold $\Sigma_{2}$, giving rise to the periodic and chaotic behaviors that were already mentioned in Sections 3.4.1 and 3.4.2, and (2) in this range, the system does not reach the 


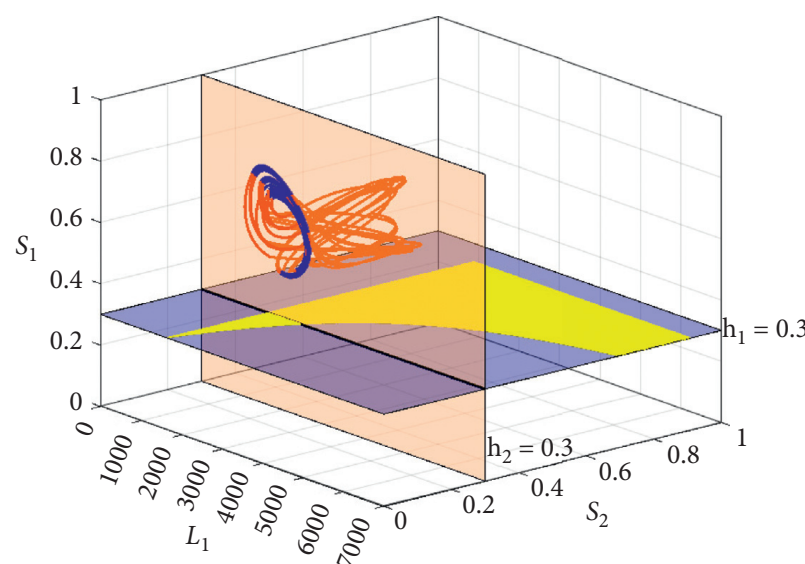

(a)

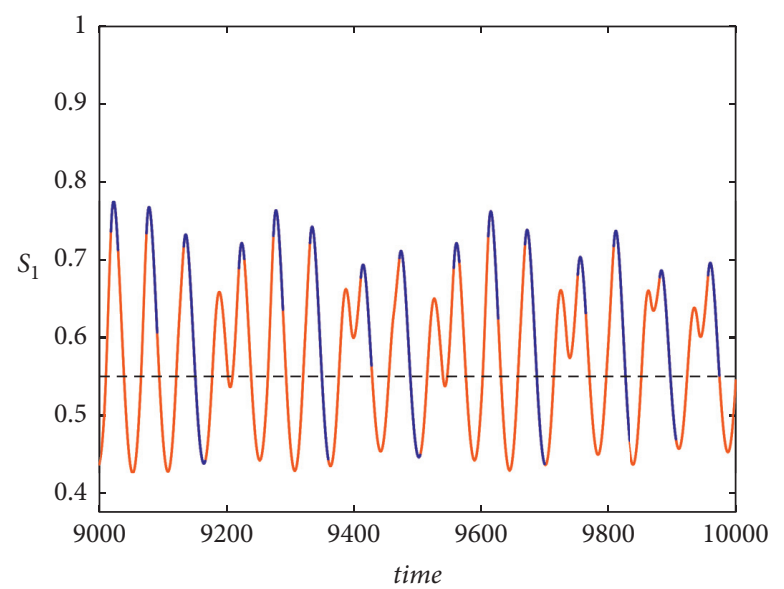

$\mathrm{C}_{2}$
--- Isolated

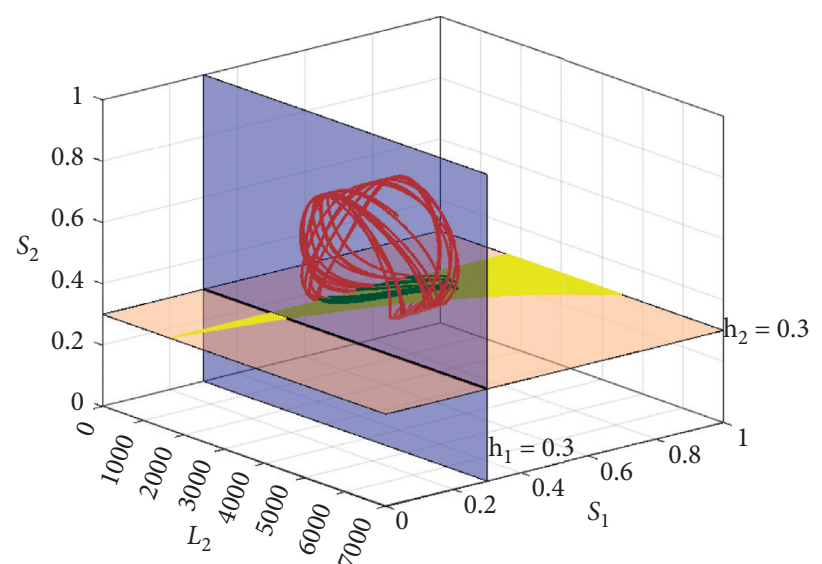

(b)

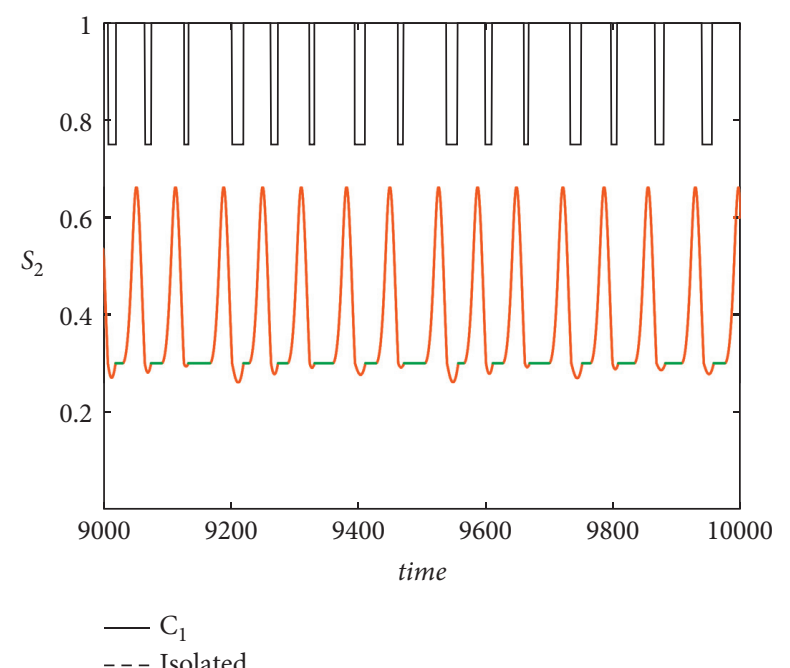

(d)

(c)

FIGURE 3: Chaotic behavior induced by switching regions for $\epsilon_{1}=5.3 e^{-5}$.

codimension two switching region. The simulations were carried out until reaching the time $t=10,000$, sampling the maximum and minimum values of the last 1000 units of time, in consideration of the changes in the behavior that the switching manifold makes on the periodic behavior of the orbits, as shown in Figure 3(d). In this way, the simulations presented in Figure 4 were obtained, where the representations of the upper steady-state, in green, map the maximums of the solution, while those of the lower part, in red, map their minima.

In this analysis, as $\epsilon_{1}$ increases in the selected range, the system shows changes in periodicity and chaos in the dynamics of the behavior of community one (population and resources), without deviating it from the surplus threshold, see Figures 4(a) and 4(c), while community two exhibits behaviors of period one for the population and periodicity and chaos for resources when they are at the deficit threshold, see Figures 4(b) and 4(d). For the final interval of the analysis, the exchange was found to occur without sliding, through orbits of period one.

\section{Discussion of Results}

In this section, the results obtained will be discussed to understand (1) the effect of the variation of the extraction capacities in an isolated community, using a two-dimensional continuous model, see Section 2, and (2) the effect of the exchange of resources between two communities, based on a Filippov system, see Section 3, considering that, to have a first approximation of sustainability as conservation of well-being in a territory, landscape, or community, it is assumed that wellbeing is having renewable resources, which, as mentioned in the introduction, oversimplifies a plausible definition of wellbeing, but allows the presentation of the possibilities of the interpretation proposed in this article for sustainability.

In the first place, regarding the effect of the variation in extraction capacities in an isolated community, it is notable that, following the bifurcation diagram in Figure 1, the dynamics of the system involves four prospective scenarios: (1) negligence: in which the extraction is insufficient to sustain the population, (2) harmony: in which the extraction 


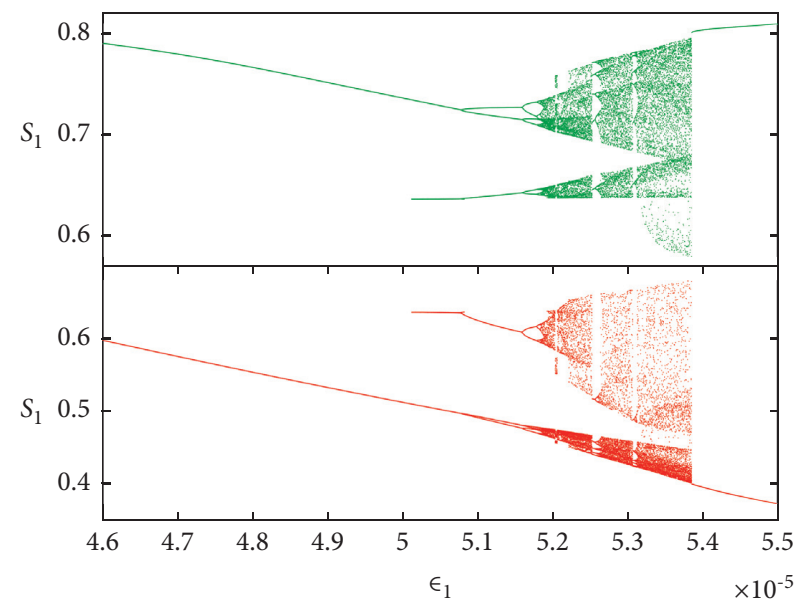

(a)

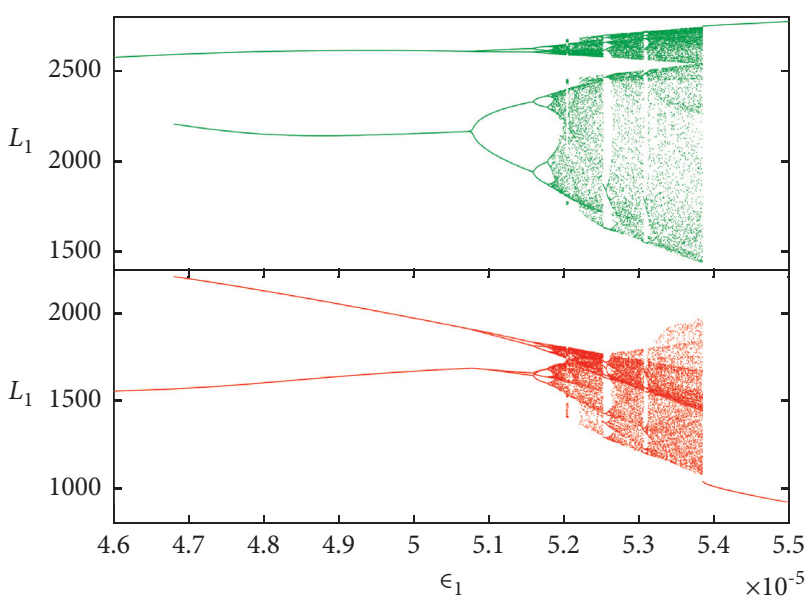

(c)

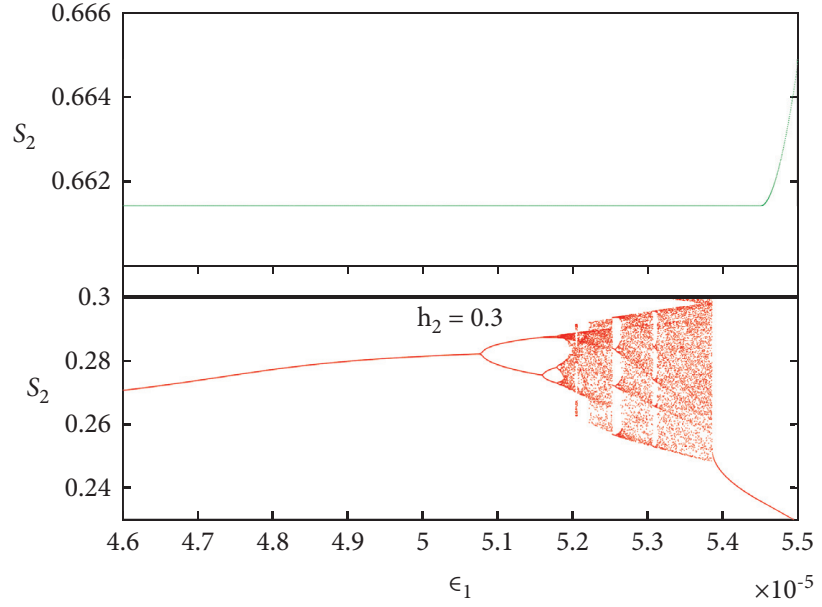

(b)

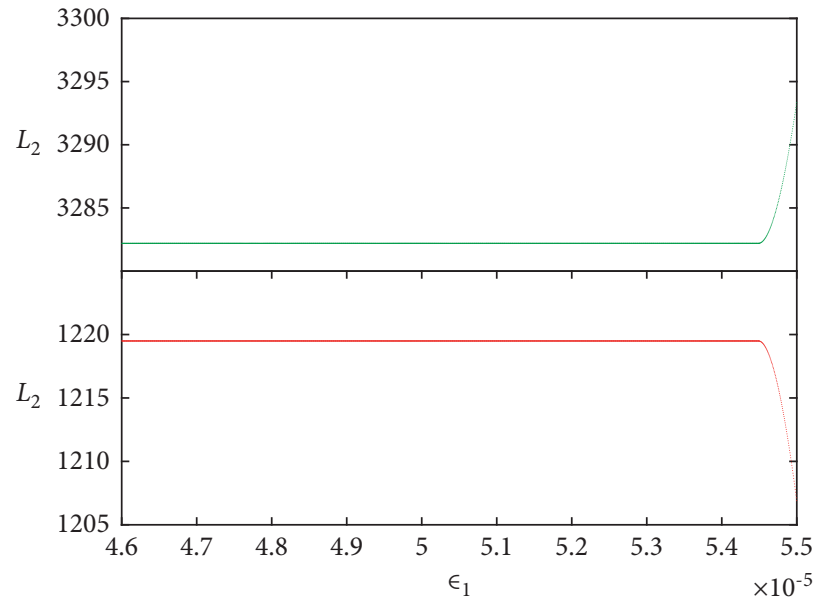

(d)

Figure 4: Steady state maps.

and recovery of resources are in equilibrium, (3) instability: in which there are continuous oscillations between scarcity and abundance, and (4) collapse: in which the excess in the extraction of resources leads to the disappearance of the population and the resources.

In this case, the conservation of well-being could only be appreciated in the harmony scenario, through the invariant set of equilibrium point type that the system defines for a certain level of extraction, so it is concluded that a territory, community, or landscape isolated, and it is only sustainable if it finds the balance between its population and the extraction of resources that it does for its maintenance, in consideration of the regenerative capacity of the resources.

This result, and its corresponding scenarios, can be compared with the current situation of humanity as a large community in a space with limited resources: planet Earth, with only the scenario that we call harmony being sustainable.

Second, on the effect of the exchange of resources between two communities based on a Filippov system, the results have allowed us to see through simulations and the dynamics emerging from the interaction between two communities that share their resources in pursuit of well-being, through invariant sets such as periodic orbits and strange attractors, who allow us to conclude that sustainability can take different invariant forms within the notion of well-being, in addition to the equilibrium form shown for an isolated community.

The reason why the exchange between the two communities reaches sustainability under these multiple geometries (equilibrium point, periodic orbit, or strange attractor), although one of the communities has been configured not to be sustainable without the exchange, is as follows: (1) there were clear conservation policies (represented through the switching manifolds) and (2) the policies had perfect control (the system was programmed deterministically). However, achieving sustainability through exchange makes the consuming community stabilize while the resource-rich community becomes unstable.

This is where an interpretation of well-being as several renewable resources fails because the instability of the stock reflects the socio-ecological instability of the one who contributes the resources to ensure that the community at the threshold of deficit can satisfy its demands for resources, 


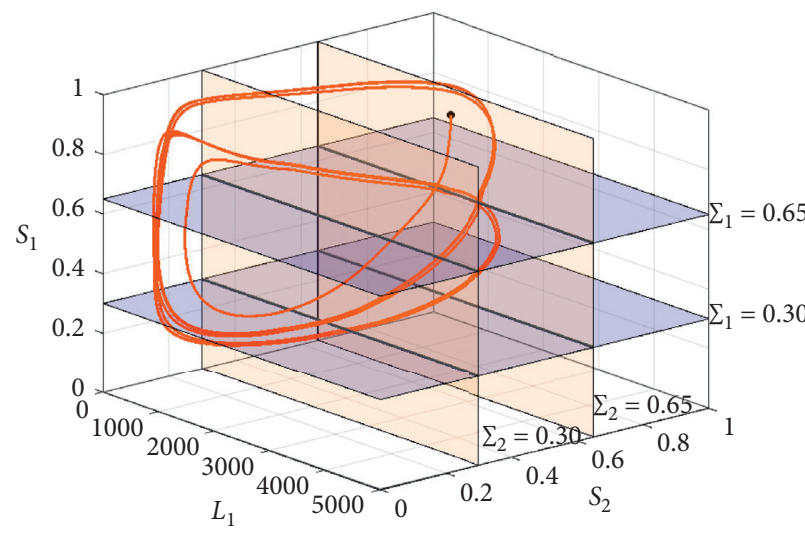

(a)

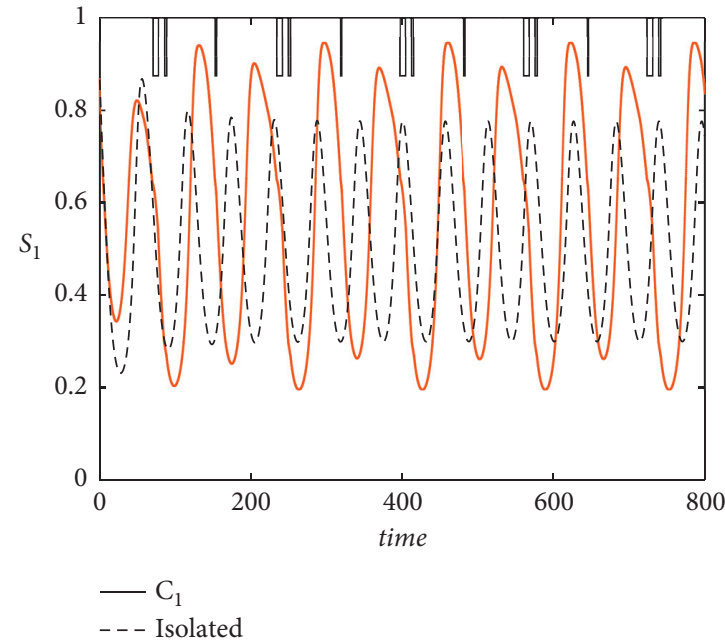

(c)

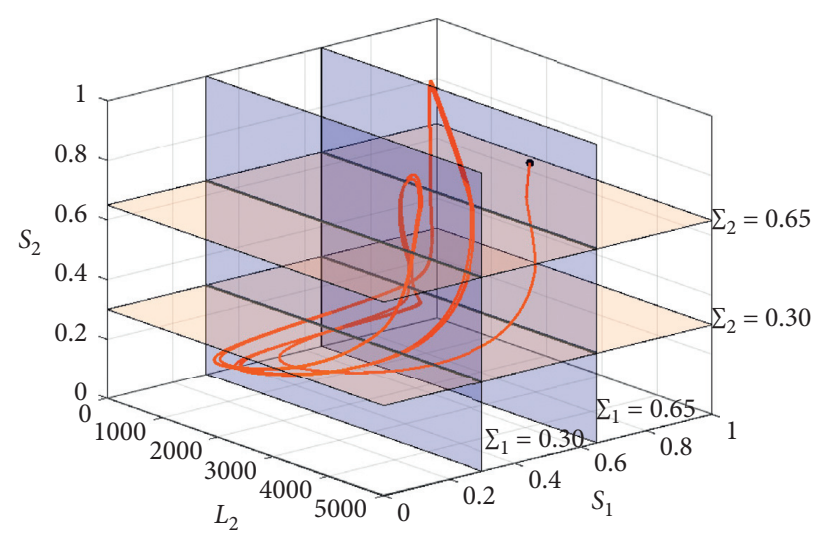

(b)

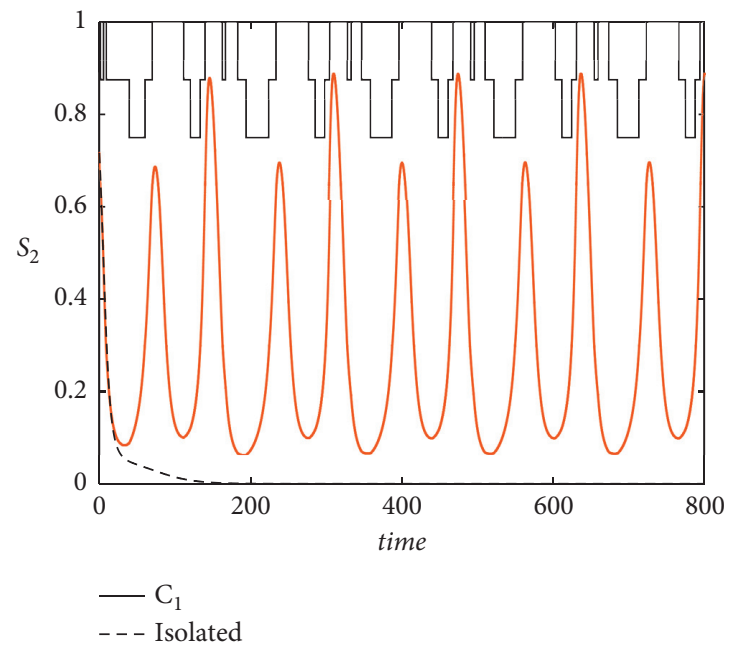

(d)

FIgURE 5: Steady state for economic cooperation between two communities when you have two switching regions. Parameter setting is $C_{i}=0.75, \sigma_{i}=0.14, \rho_{1}=0.03, \rho_{2}=0.04, T_{i}=700, K_{i}=12000, \beta_{1}=5.8 e^{-5}, \epsilon_{2}=7 e^{-5}$, and $y \phi_{i}=0.4$.

without questioning or regulating the needs of the extractivist community, meaning that the contributing community maintains the stability of the relationship, despite the stability of its other socio-ecological dimensions. In other words, satisfying the needs of an extractive community despite another community can generate socio-ecological instabilities in the community that it contributes (violence, tyrannies, wage abuses, inhumane living conditions, etc.?). So, speaking of sustainability, the definition of what is well-being must be multidimensional, involving at least people, animals (domestic or livestock), and ecosystems.

About sliding, it shows how a community is trapped in a set of transition states between one threshold and another while regulating the population. This can be derived from a biological impulse of humanity that, without clear controls, tends to deteriorate its well-being, falling into the tragedy of commons ground.

The values of the exchange constants, together with the values of the extraction rates, proved to be conditioning factors for the development or deterioration of the communities, suggesting that they should be careful with their definition, before proclaiming a national or international exchange policy.

\section{Conclusions}

The exchange avoids collapse and leads to a process of recovery of the community in deficit, which is not immediate, since it must overcome a delay that occurs while sliding over the switching manifold, possibly related to the recovery of its resources and education for cultural transformation and sustainable management, to finally appreciate recovery and stop dependence on the other community.

The approach made in this paper from mathematical modeling for the analysis of the sustainability of territories, communities, and landscapes, between which there may or may not be exchanges, understanding that sustainability occurs when well-being is a law of conservation of the spatial unit of analysis, allows to understand that 
(i) Without exchange possibilities, a population will depend on the extraction rate and the regeneration capacity of its resources, to prevail or disappear.

(ii) The exchange of resources is key to the survival of the human species, without this implying sustainability. This cooperation uses redundancy in the other to maintain itself.

(iii) A definition of well-being limited to the number of resources as a need can generate undesirable instabilities in the contributing socio-ecological systems unless that is the sustainability that we wish to establish.

(iv) Sustainability can be visualized in the attraction basins of the invariant sets of socio-ecological systems whose states do not include null values, so it can be said that there are sustainabilities and that sustainability analyzes have many opportunities from modeling and analysis with Filippov systems and bifurcation theory.

\section{Future Research}

The coupling introduces two new parameters into the system, $h_{i}$ and $C_{i}$. As future work, it is proposed to evaluate the effect that varying one of the two has on the exchange dynamics, given that the change of the switching manifold or the exchange values, would allow evaluating which would be the threshold that leads to the best performance of the system, even if those values are not equal.

It is also proposed to consider the existence of more than one switching region for the definition of the decision levels of the system, as proposed in Figure 5.

Finally, it is proposed to establish sets of needs that allow defining in a multidimensional way the well-being of the territory, community, or landscape and promoting socioecological arrangements that allow us not only to have life but to have it with dignity.

\section{Data Availability}

The data used to support the findings of the study are available from the corresponding author upon request.

\section{Conflicts of Interest}

The authors declare that there are no conflicts of interest regarding the study of this paper.

\section{Acknowledgments}

Christian Erazo acknowledges support from University Antonio Nariño through the Research Project 2018216.

\section{References}

[1] G. H. Brundtland, Our Common FutureOxford University Press, Oxford, UK, 1987.
[2] D. Meadows, "Limits to growth, a report for the club of Rome's project on the predicament of mankind," Universe Books, Chelsea Green Publishing, Hartford, VT, USA, 1972.

[3] R. K. Turner, "Sustainability: principles and practice," in Sustainable Environmental Economics and Management: Principles and Practice, R. K. Turner, Ed., pp. 3-36, Belhaven Press, London, UK, 1993.

[4] J. Wu, "Landscape sustainability science: ecosystem services and human well-being in changing landscapes," Landscape Ecology, vol. 28, no. 6, pp. 999-1023, 2013.

[5] J. Elkington, "Enter the triple bottom line," in The Triple Bottom Line: Does it All Add up? Assessing the Sustainability of Business and CSR, A. Henriques and J. Richardson, Eds., pp. 1-186, Earthscan, London, UK, 2004.

[6] J. M. Redondo, "Landscape sustainability analysis: methodological approach from dynamical systems," in Journal of Physics: Conference SeriesIOP Publishing, Bristol, UK, 2019.

[7] A. F. Filippov, "Differential equations with discontinuous right-hand side," American Mathematical Society Translations, vol. 42, no. 2, pp. 354-362, 1964.

[8] A. F. Filippov, "Equations with the right-hand side continuous in $x$ and discontinuous in $t$," in Differential Equations with Discontinuous Righthand Sides, pp. 3-47, Springer, Berlin, Germany, 1988.

[9] M. A. Teixeira, "Stability conditions for discontinuous vector fields," Journal of Differential Equations, vol. 88, no. 1, pp. 15-29, 1990.

[10] Y. A. Kuznetsov, S. Rinaldi, and A. Gragnani, "One-parameter bifurcations in planar Filippov systems," International Journal of Bifurcation and Chaos, vol. 13, no. 8, pp. 2157-2188, 2003.

[11] M. Guardia, T. M. Seara, and M. A. Teixeira, "Generic bifurcations of low codimension of planar Filippov systems," Journal of Differential Equations, vol. 250, no. 4, pp. 19672023, 2011.

[12] I. Remco, H. Nijmeijer, Dynamics and Bifurcations of Nonsmooth Mechanical Systems, Vol. 18, Springer Science \& Business Media, Berlin, Germany, 2013.

[13] M. Di Bernardo, C. J. Budd, A. R. Champneys et al., "Bifurcations in nonsmooth dynamical systems," SIAM Review, vol. 50, no. 4, pp. 629-701, 2008.

[14] V. Utkin, "Variable structure systems with sliding modes," IEEE Transactions on Automatic Control, vol. 22, no. 2, pp. 212-222, 1977.

[15] E. Freire, E. Ponce, F. Rodrigo, and F. Torres, "Bifurcation sets of continuous piecewise linear systems with two zones," International Journal of Bifurcation and Chaos, vol. 8, no. 11, pp. 2073-2097, 1998.

[16] V. Carmona, E. Freire, E. Ponce, and F. Torres, "On simplifying and classifying piecewise-linear systems," IEEE Transactions on Circuits and Systems I: Fundamental Theory and Applications, vol. 49, no. 5, pp. 609-620, 2002.

[17] E. Ponce, J. Ros, and E. Vela, "Limit cycle and boundary equilibrium bifurcations in continuous planar piecewise linear systems," International Journal of Bifurcation and Chaos, vol. 25, no. 3, Article ID 1530008, 2015.

[18] J. Llibre, E. Ponce, and J. Ros, "Algebraic determination of limit cycles in a family of three-dimensional piecewise linear differential systems," Nonlinear Analysis: Theory, Methods \& Applications, vol. 74, no. 17, pp. 6712-6727, 2011.

[19] B. R. de Freitas, J. Llibre, and J. C. Medrado, "Limit cycles of continuous and discontinuous piecewise-linear differential systems in R3," Journal of Computational and Applied Mathematics, vol. 338, pp. 311-323, 2018. 
[20] R. Cristiano and D. J. Pagano, "Two-parameter boundary equilibrium bifurcations in 3D-filippov systems," Journal of Nonlinear Science, vol. 29, no. 6, pp. 2845-2875, 2019.

[21] O. Makarenkov and J. S. W. Lamb, "Dynamics and bifurcations of nonsmooth systems: a survey," Physica D: Nonlinear Phenomena, vol. 241, no. 22, pp. 1826-1844, 2012.

[22] A. Colombo, M. di Bernardo, S. J. Hogan, and M. R. Jeffrey, "Bifurcations of piecewise smooth flows: perspectives, methodologies and open problems," Physica D: Nonlinear Phenomena, vol. 241, no. 22, pp. 1845-1860, 2012.

[23] F. Dercole, F. Della Rossa, A. Colombo, and Y. A. Kuznetsov, "Two degenerate boundary equilibrium bifurcations in planar Filippov systems," SIAM Journal on Applied Dynamical Systems, vol. 10, no. 4, pp. 1525-1553, 2011.

[24] M. R. Jeffrey and A. Colombo, "The two-fold singularity of discontinuous vector fields," SIAM Journal on Applied Dynamical Systems, vol. 8, no. 2, pp. 624-640, 2009.

[25] M. R. Jeffrey and S. J. Hogan, "The geometry of generic sliding bifurcations," SIAM Review, vol. 53, no. 3, pp. 505-525, 2011.

[26] M. R. Jeffrey, "Nondeterminism in the limit of nonsmooth dynamics," Physical Review Letters, vol. 106, no. 25, Article ID 254103, 2011.

[27] Z. Du, Y. Li, and W. Zhang, "Bifurcation of periodic orbits in a class of planar Filippov systems," Nonlinear Analysis: Theory, Methods \& Applications, vol. 69, no. 10, pp. 3610-3628, 2008.

[28] Z. Du and Y. Li, "Bifurcation of periodic orbits with multiple crossings in a class of planar Filippov systems," Mathematical and Computer Modelling, vol. 55, no. 3-4, pp. 1072-1082, 2012.

[29] B. N. Arne, "Non-periodic motion caused by grazing incidence in an impact oscillator," Journal of Sound and Vibration, vol. 145, pp. 279-297, 1991.

[30] M. Di Bernardo, P. Kowalczyk, and A. Nordmark, "Bifurcations of dynamical systems with sliding: derivation of normal-form mappings," Physica D: Nonlinear Phenomena, vol. 170, no. 3-4, pp. 175-205, 2002.

[31] M. Di Bernardo, P. Kowalczyk, and A. Nordmark, "Sliding bifurcations: a novel mechanism for the sudden onset of chaos in dry friction oscillators," International Journal of Bifurcation and Chaos, vol. 13, no. 10, pp. 2935-2948, 2003.

[32] D. J. W. Simpson, "A compendium of Hopf-like bifurcations in piecewise-smooth dynamical systems," Physics Letters A, vol. 382, no. 35, pp. 2439-2444, 2018.

[33] L. Dieci and L. Lopez, "Sliding motion in Filippov differential systems: theoretical results and a computational approach," SIAM Journal on Numerical Analysis, vol. 47, no. 3, pp. 2023-2051, 2009.

[34] L. Dieci and L. Lopez, "A survey of numerical methods for IVPs of ODEs with discontinuous right-hand side," Journal of Computational and Applied Mathematics, vol. 236, no. 16, pp. 3967-3991, 2012.

[35] D. J. W. Simpson, "On resolving singularities of piecewisesmooth discontinuous vector fields via small perturbations," Discrete \& Continuous Dynamical Systems-A, vol. 34, no. 9, pp. 3803-3830, 2014.

[36] D. J. W. Simpson and R. Kuske, "Stochastic perturbations of periodic orbits with sliding," Journal of Nonlinear Science, vol. 25, no. 4, pp. 967-1014, 2015.

[37] C. Erazo, M. E. Homer, P. T. Piiroinen, and M. Di Bernardo, "Dynamic cell mapping algorithm for computing basins of attraction in planar Filippov systems," International Journal of Bifurcation and Chaos, vol. 27, no. 12, Article ID 1730041, 2017.
[38] E. Bossolini, M. Brns, and K. U. Kristiansen, "Canards in stiction: on solutions of a friction oscillator by regularization," SIAM Journal on Applied Dynamical Systems, vol. 16, no. 4, pp. 2233-2258, 2017.

[39] E. Blokhina, D. Galayko, D. Fournier-Prunaret, and O. Feely, "Sliding in a piecewise-smooth dynamical system with a holdon effect," Physics Letters A, vol. 378, no. 42, pp. 3085-3092, 2014.

[40] M. Guardia, S. J. Hogan, and T. M. Seara, "An analytical approach to codimension-2 sliding bifurcations in the dryfriction oscillator," SIAM Journal on Applied Dynamical Systems, vol. 9, no. 3, pp. 769-798, 2010.

[41] P. Kowalczyk and P. T. Piiroinen, "Two-parameter sliding bifurcations of periodic solutions in a dry-friction oscillator," Physica D: Nonlinear Phenomena, vol. 237, no. 8, pp. 10531073, 2008.

[42] A. Pratap, "Finite-time synchronization criterion of graph theory perspective fractional-order coupled discontinuous neural networks," Advances in Difference Equations, vol. 2020, p. 1, 2020.

[43] A. Pratap, "Quasi-pinning synchronization and stabilization of fractional order BAM neural networks with delays and discontinuous neuron activations," Chaos, Solitons and Fractals, vol. 131, 2020.

[44] A. Pratap, R. Raja, J. Cao, C. P. Lim, and O. Bagdasar, "Stability and pinning synchronization analysis of fractional order delayed Cohen-Grossberg neural networks with discontinuous activations," Applied Mathematics and Computation, vol. 359, pp. 241-260, 2019.

[45] X. Yang, Q. Song, J. Liang, and B. He, "Finite-time synchronization of coupled discontinuous neural networks with mixed delays and nonidentical perturbations," Journal of the Franklin Institute, vol. 352, no. 10, pp. 4382-4406, 2015.

[46] M. Forti and P. Nistri, "Global convergence of neural networks with discontinuous neuron activations," IEEE Transactions on Circuits and Systems I: Fundamental Theory and Applications, vol. 50, no. 11, pp. 1421-1435, 2003.

[47] C. Chen, "Finite-time Mittag-Leffler synchronization of fractional-order delayed memristive neural networks with parameters uncertainty and discontinuous activation functions," Chinese Physics B, vol. 29, p. 4, 2020.

[48] S. Liu, Y. Yu, S. Zhang, and Y. Zhang, "Robust stability of fractional-order memristor-based Hopfield neural networks with parameter disturbances," Physica A: Statistical Mechanics and Its Applications, vol. 9, pp. 845-854, 2018.

[49] A. Abdurahman, H. Jiang, and C. Hu, "General decay synchronization of memristor-based Cohen-Grossberg neural networks with mixed time-delays and discontinuous activations," Journal of the Franklin Institute, vol. 354, no. 15, pp. 7028-7052, 2017.

[50] Z. Cai, L. Huang, M. Zhu, and D. Wang, "Finite-time stabilization control of memristor-based neural networks," Nonlinear Analysis: Hybrid Systems, vol. 20, no. 37, pp. 37-54, 2016.

[51] Y. Gu, Y. Yu, and H. Wang, "Synchronization for fractionalorder time-delayed memristor-based neural networks with parameter uncertainty," Journal of the Franklin Institute, vol. 353, no. 15, pp. 3657-3684, 2016.

[52] H. Bao, J. H. Park, and J. Cao, "Matrix measure strategies for exponential synchronization and anti-synchronization of memristor-based neural networks with time-varying delays," Applied Mathematics and Computation, vol. 270, pp. 543-556, 2015. 
[53] H. Wu, R. Li, S. Ding, X. Zhang, and R. Yao, "Complete periodic adaptive antisynchronization of memristor-based neural networks with mixed time-varying delays," Canadian Journal of Physics, vol. 92, no. 11, pp. 1337-1349, 2014.

[54] Z. Cai and L. Huang, "Lyapunov-Krasovskii stability analysis of delayed Filippov system: applications to neural networks with switching control," International Journal of Robust and Nonlinear Control, vol. 30, no. 2, pp. 699-718, 2020.

[55] Z. Cai, L. Huang, and L. Zhang, "Improved switching controllers for finite-time synchronization of delayed neural networks with discontinuous activations," Journal of the Franklin Institute, vol. 354, no. 15, pp. 6692-6723, 2017.

[56] Z. Cai, L. Huang, D. Wang, and L. Zhang, "Periodic synchronization in delayed memristive neural networks based on Filippov systems," Journal of the Franklin Institute, vol. 352, no. 10, pp. 4638-4663, 2015.

[57] H. Wu, "Robust almost periodic dynamics for interval neural networks with mixed time-varying delays and discontinuous activation functions," Abstract and Applied Analysis, vol. 2013, Article ID 630623, 13 pages, 2013.

[58] J. A. Taborda, F. Angulo, and G. Olivar, "Bifurcation analysis on nonsmooth torus destruction scenario of delayed-pwm switched buck converter," International Journal of Bifurcation and Chaos, vol. 19, no. 7, pp. 2193-2212, 2009.

[59] A. Jorge, G. Olivar, and F. Angulo, "Smooth and Filippov models of sustainable development: bifurcations and numerical computations," Differential Equations and Dynamical Systems, vol. 21, pp. 173-184, 2013.

[60] O. Valencia-Rodríguez, G. Olivar-Tost, and J. M. Redondo, "Modeling a productive system incorporating elements of business sustainability," Dyna, vol. 85, no. 207, pp. 113-122, 2018.

[61] J. Valencia-Calvo, G. Olivar-Tost, J. D. Morcillo-Bastidas, C. J. Franco-Cardona, and I. Dyner, "Non-smooth dynamics in energy market models: a complex approximation from system dynamics and dynamical systems approach," IEEE Access, vol. 8, pp. 128877-128896, 2020.

[62] J. M. Redondo, "Making decisions in national energy markets with bifurcation analysis," in Journal of Physics: Conference SeriesInstitute of Physics Publishing, Bristol, UK, 2019.

[63] J. M. Redondo, "Modeling for the regional integration of electricity markets," Energy for Sustainable Development, vol. 43, pp. 100-113, 2018.

[64] J. Valencia-Calvo, C. J. Franco-Cardona, G. Olivar-Tost, and I. Dyner-Rezonzew, "Enfoque metodológico para el estudio y representación de comportamientos complejos en mercados de electricidad," Ingeniería y Ciencia, vol. 12, no. 24, pp. 195-220, 2016.

[65] C. Chen, C. Li, and Y. Kang, "Modelling the effects of cutting off infected branches and replanting on fire-blight transmission using Filippov systems," Journal of Theoretical Biology, vol. 439, pp. 127-140, 2018.

[66] G. Tang, W. Qin, and S. Tang, "Complex dynamics and switching transients in periodically forced Filippov preypredator system," Chaos, Solitons \& Fractals, vol. 61, pp. 13-23, 2014.

[67] S. Tang, J. Liang, Y. Xiao, and R. A. Cheke, "Sliding bifurcations of Filippov two stage pest control models with economic thresholds," SIAM Journal on Applied Mathematics, vol. 72, no. 4, pp. 1061-1080, 2012.

[68] B. Tang, Y. Xiao, R. A. Cheke, and N. Wang, "Piecewise virusimmune dynamic model with HIV-1 RNA-guided therapy," Journal of Theoretical Biology, vol. 377, pp. 36-46, 2015.
[69] B. Tang, Y. Xiao, S. Sivaloganathan, and J. Wu, “A piecewise model of virus-immune system with effector cell-guided therapy," Applied Mathematical Modelling, vol. 47, pp. 227248, 2017.

[70] F. Dercole, A. Gragnani, and S. Rinaldi, "Bifurcation analysis of piecewise smooth ecological models," Theoretical Population Biology, vol. 72, no. 2, pp. 197-213, 2007.

[71] X. Yang, Z. Yang, and X. Nie, "Exponential synchronization of discontinuous chaotic systems via delayed impulsive control and its application to secure communication," Communications in Nonlinear Science and Numerical Simulation, vol. 19, no. 5, pp. 1529-1543, 2014.

[72] J. Amador-Moncada, "Dinámicas no-lineales y no-suaves en procesos estrés-enfermedad/nonlinear and nonsmooth dynamics in stress-sickness processes," Ciencia en Desarrollo, vol. 8, no. 1, pp. 9-19, 2017.

[73] F. Dercole and Y. A. Kuznetsov, "SlideCont: an auto 97 driver for bifurcation analysis of Filippov systems," ACM Transactions on Mathematical Software, vol. 1, no. 1, pp. 95-119, 2005.

[74] P. T. Piiroinen and Y. A. Kuznetsov, "An event-driven method to simulate Filippov systems with accurate computing of sliding motions," ACM Transactions on Mathematical Software, vol. 34, no. 3, 2008.

[75] P. Thota and H. Dankowicz, "TC-HAT (widehat): a novel toolbox for the continuation of periodic trajectories in hybrid dynamical systems," SIAM Journal on Applied Dynamical Systems, vol. 7, no. 4, pp. 1283-1322, 2008.

[76] G. Formica, "Coupling FEM with parameter continuation for analysis of bifurcations of periodic responses in nonlinear structures," Journal of Computational and Nonlinear Dynamics, vol. 8, no. 2, 2013.

[77] H. Dankowicz, Multibody Mechanics and Visualization, Springer Science \& Business Media, Berlin, Germany, 2005.

[78] J. Brander and M. Scott Taylor, "The simple economics of Easter island: a Ricardo-Malthus model of renewable resource use," American Economic Review, vol. 88, no. 1, pp. 119-138, 1998.

[79] R. Reuveny and C. S. Decker, "Easter island: historical anecdote or warning for the future?" Ecological Economics, vol. 35, no. 2, pp. 271-287, 2000.

[80] S. D’Alessandro, "Non-linear dynamics of population and natural resources: the emergence of different patterns of development," Ecological Economics, vol. 62, no. 3, pp. 473-481, 2007.

[81] T. R. Dalton and R. Morris Coats, "Could institutional reform have saved Easter island?" Journal of Evolutionary Economics, vol. 10, no. 5, pp. 489-505, 2000.

[82] T. R. Dalton, R. M. Coats, and B. R. Asrabadi, "Renewable resources, property-rights regimes and endogenous growth," Ecological Economics, vol. 52, no. 1, pp. 31-41, 2005.

[83] J. C. V. Pezzey and J. M. Anderies, "The effect of subsistence on collapse and institutional adaptation in population-resource societies," Journal of Development Economics, vol. 72, no. 1, pp. 299-320, 2003.

[84] J. M. Anderies and M. Hegmon, "Robustness and resilience across scales: migration and resource degradation in the prehistoric U.S. southwest," Ecology and Society, vol. 16, no. 2, pp. 1708-3087, 2011.

[85] S. Roman, S. Bullock, and M. Brede, "Coupled societies are more robust against collapse: a hypothetical look at Easter island," Ecological Economics, vol. 132, pp. 264-278, 2017.

[86] D. Angulo, F. Angulo, and G. Olivar, "Dynamics and forecast in a simple model of sustainable development for rural 
populations," Bulletin of Mathematical Biology, vol. 77, no. 2, pp. 368-389, 2015.

[87] Z. Dockstader, C. T. Bauch, and M. Anand, "Interconnections accelerate collapse in a socio-ecological metapopulation," Sustainability, vol. 11, pp. 2071-1050, 2019.

[88] M. B. Eppinga, K. Siteur, M. Baudena et al., "Long-term transients help explain regime shifts in consumer-renewable resource systems," Communications Earth \& Environment, vol. 2, no. 1, 2021.

[89] Y. A. Kuznetsov, Elements of Applied Bifurcation Theory, Vol. 112, Springer Science \& Business Media, Berlin, Germany, 2013.

[90] A. Dhooge, W. Govaerts, and Y. A. Kuznetsov, "Matcont," ACM Transactions on Mathematical Software, vol. 29, no. 2, pp. 141-164, 2003.

[91] J. C. Alexander and I. S. Thomas, "Sliding modes in intersecting switching surfaces, I: blending," Houston Journal of Mathematics, vol. 24, no. 3, pp. 545-569, 1998.

[92] L. Dieci and F. Difonzo, "The moments sliding vector field on the intersection of two manifolds," Journal of Dynamics and Differential Equations, vol. 29, no. 1, pp. 169-201, 2017.

[93] M. R. Jeffrey, "Hidden dynamics in models of discontinuity and switching," Physica D: Nonlinear Phenomena, vol. 273274, pp. 34-45, 2014. 